VoL. 47 (1993) [407-414]

\title{
ON A WEAK* DROP PROPERTY
}

\author{
WARREN B. MOORS
}

\begin{abstract}
Rolewicz said that the norm of a Banach space $(X,\|\cdot\|)$ has the drop property if for every closed set $C$ disjoint from the closed unit ball $B(X)$, there exists an $\approx \in C$ such that $\operatorname{co}(x, B(X)) \cap C=\{x\}$. He then showed that if the norm of a Banach space $(X,\|\cdot\|)$ has the drop property then it is reflexive. Later, in 1990 Giles, Sims and Yorke showed that the norm of Banach space $(X,\|\cdot\|)$ has the drop property if and only if the duality mapping $f \rightarrow D(f)$ from the dual sphere $S\left(X^{*}\right)$ into subsets of the second dual sphere $S\left(X^{* *}\right)$ is norm upper semi-continuous and norm compact-valued on $S\left(X^{*}\right)$.

In this paper we investigate a geometric condition similar to the drop property which is characterised by the duality mapping $x \rightarrow D(x)$ from $S(X)$ into subsets of $S\left(X^{*}\right)$ being norm upper semi-continuous and norm compact-valued.
\end{abstract}

\section{INTRODUCTION}

Given a Banach space $(X,\|\cdot\|)$ over the real numbers, with dual $X^{*}$, we denote the closed unit ball of $X$ by $B(X) \equiv\{x \in X:\|x\| \leqslant 1\}$ and the unit sphere of $X$ by $S(X) \equiv\{x \in X:\|x\|=1\}$. By a drop induced by a point $x \notin B(X)$ we mean the closed set $D(x, B(X))$; which is the convex hull of $x$ and $B(X)$.

Given $f \in S\left(X^{*}\right)$ and $\delta>0$, the slice of $B(X)$ defined by $f$ and $\delta$ is the subset: $S(B(X), f, \delta) \equiv\{x \in B(X): f(x)>1-\delta\}$. For each $x \in S(X)$ we denote by $D(x)$ the set of $\left\{f \in S\left(X^{*}\right): f(x)=1\right\}$. The set-valued mapping $x \rightarrow D(x)$ from $S(X)$ into subsets of $S\left(X^{*}\right)$ is called the duality mapping on $S(X)$.

In 1972 Danes̃ [2] proved the following seminal result; which has since become known as the drop theorem.

Theorem 1.1. Let $C$ be a closed subset of a Banach space such that $\inf \{\|x\|: x \in C\}>1$. Then there is a point $x \in C$ such that $D(x, B(X)) \cap C=\{x\}$.

Later in 1987, Danes' drop theorem motivated Rolewicz [7] to consider the following geometrical condition. He said that the norm $\|\cdot\|$ on a Banach space has the drop property if for every closed set $C$ disjoint from the closed unit ball $B(X)$, there exists an $x \in C$ such that $D(x, B(X)) \cap C=\{x\}$.

Received 11 May 1992

Copyright Clearance Centre, Inc. Serial-fee code: 0004-9729/93 \$A2.00+0.00. 
In that paper Rolewicz established a connection between the drop property and Kuratowski's index of non-compactness, which he then used to show that every Banach space which admits an equivalent norm with the drop property is reflexive.

For the reader's convenience, we recall the definition and elementary properties sataisfied by Kuratowski's index of non-compactness, [1, p.4].

For a bounded set $E$ in a metric space $(X, d)$ the Kuratowski index of noncompactness of $E$ is:

$\alpha(E) \equiv \inf \{r>0$ : is covered by a finite family of sets of diameter less than $r\}$.

The following elementary propositions are stated without proof.

Proposition 1.2. For a Banach space $(X,\|\cdot\|)$, Kuratowski's index of noncompactness satisfies the following properties for bounded sets on $X$.

1. $\alpha(E) \geqslant 0$ for $E \subseteq X$.

2. $\alpha(E) \leqslant \operatorname{diam} E$ for $E \subseteq X$.

3. $\alpha(E) \leqslant \alpha(F)$ if $E \subseteq F \subseteq X$.

4. $\alpha(E)=\alpha(E)$ for $E \subseteq X$ where $\bar{E}$ is the closure of $E$ in $X$.

5. $\alpha\left(\bigcup_{j=1}^{n} E_{j}\right)=\max \left\{\alpha\left(E_{j}\right): j \in\{1,2, \ldots, n\}\right\}$, for $E_{j} \subseteq X$ for all $j \in$ $\{1,2, \ldots, n\}$.

6. $\alpha(E \cap F) \leqslant \min \{\alpha(E), \alpha(F)\}$ for $E, F \subseteq X$.

7. $\alpha(E)=0$ if and only if $E$ is a totally bounded subset of $X$.

8. $\alpha(E+F) \leqslant \alpha(E)+\alpha(F)$ for $E, F \subseteq X$.

9. $\alpha(k E)=|k| \alpha(E)$ for $E \subseteq X$ and $k \in \mathbb{R}$.

10. $\alpha(c o E)=\alpha(E)$ for $E \subseteq X$, where coE denotes the convex hull of $E$ in $X$.

Proposition 1.3. Let $(X, d)$ be a metric space, and $E$ a non-empty subset of $X$.

1. If $E$ contains a sequence $\left\{x_{n}\right\}_{n=1}^{\infty}$ such that $d\left(x_{n}, x_{m}\right) \geqslant \varepsilon>0$ whenever $m \neq n$, then $\alpha(E) \geqslant \varepsilon$.

2. If $\alpha(E)>2 \varepsilon>0$ then there exists a sequence $\left\{x_{n}\right\}_{n=1}^{\infty}$ contained in $E$ such that $d\left(x_{n}, x_{m}\right)>\varepsilon$ whenever $m \neq n$.

Now, since the pioneering work of Rolewicz many people have contributed to the development of the drop property and several variations upon Rolewicz's original definition have been considered. However of particular interest to us is the recent work of Giles and Moors [4]. The above authors introduced a new continuity condition related to Kuratowski's index of non-compactness. They said that a set-valued mapping $\Phi$ from a topological space $A$ into subsets of a metric space is $\alpha$ upper semi-continuous at $t$ in 
$A$ if given $\varepsilon>0$ there exists an open neighbourhood $U$ of $t$ such that $\alpha(\Phi(U))<\varepsilon$. Using this definition they showed that the duality mapping from the unit sphere of a Banach space $X$ is $\alpha$ upper semi-continuous at a point $x$ if and only if the duality mapping is norm upper semi-continuous and norm compact valued at $x \in S(X)$. This result, along with the fact that a Banach space $X$ has the drop property if and only if the duality mapping $x \rightarrow D(x)$ from $S\left(X^{*}\right)$ into subsets of $S\left(X^{* *}\right)$ is norm upper semi-continuous and norm compact valued on $S\left(X^{*}\right)$, [3, Corollary 4], enabled Giles and Moors to deduce the following theorem, [4, Corollary 3.3].

THEOREM 1.4. A Banach space $(X,\|\cdot\|)$ has the drop property if and only if the duality mapping $f \rightarrow D(f)$ from $S\left(X^{*}\right)$ into subsets of $S\left(X^{* *}\right)$ is a upper semicontinuous on $S\left(X^{*}\right)$.

In this paper we consider a geometric property possessed by the dual of a Banach space, called the weak $k^{*}$ drop property, and we show that this property is characterised by the duality mapping from the sphere in $X$ into subsets of $S\left(X^{*}\right)$, being $\alpha$ upper semi-continuous on $S(X)$, or equivalently, by the duality mapping being norm upper semi-continuous and norm compact valued on $S(X)$.

Finally, we establish the relationship between the drop property and the weak * drop property, and obtain a new result about spaces which possess the drop property.

We end this section with the following definitions.

For a continuous convex function $\phi$ on an open convex subset $A$ of a Banach space $X$, a subgradient of $\phi$ at $x \in A$ is a continuous linear functional $f$ on $X$ such that $f(y-x) \leqslant \phi(y)-\phi(x)$ for all $y$ in $A$. The subdifferential of $\phi$ at $x$, denoted by $\partial \phi(x)$, is the set of all subgradients of $\phi$ at $x$, and is non-empty for each $x \in A$.

\section{Characterisations of the Weak* Drop Property}

We begin this section with the following consequence [6, Proposition 4.1] of the Brøndsted-Rockafellar Theorem.

Proposition 2.1. Consider a weak ${ }^{*}$ compact convex set $E$ with $0 \in E$ in the dual of a Banach space $X$. Define the functional $p$ on $X$ by $p(x) \equiv \max \{f(x): f \in E\}$. Then $p$ is a continuous sublinear functional, and for every $x \in X \backslash\{0\}$ and $\delta>0$, $\overline{S\left(E, \widehat{x}, \delta^{2}\right)} \subseteq \partial p(B(x, \delta))+\delta B\left(X^{*}\right)$.

The unit ball $B(X)$ on a Banach space $X$ has property $\alpha$ for $f \in S\left(X^{*}\right)$ if for each $\varepsilon>0$ there exists a $\delta>0$ such that $\alpha(S(B(X), f, \delta))<\varepsilon$. Furthermore, we say that the dual ball $B\left(X^{*}\right)$ has weak* property $\alpha$ if $B\left(X^{*}\right)$ has property $\alpha$ for each $\widehat{x} \in S(\widehat{X})$.

Proposition 2.2. Consider the Banach space $(X,\|\cdot\|)$. The duality mapping $x \rightarrow D(x)$ from $S(X)$ into subsets of $S\left(X^{*}\right)$ is a upper semi-continuous at $x \in S(X)$ 
if and only if $B\left(X^{*}\right)$ has property $\alpha$ for $x \in S(X)$.

Proof: Firstly, we note that $D=\left.(\partial\|\cdot\|)\right|_{S(X)}[4$, Theorem 3.5] and that the duality mapping $x \rightarrow D(x)$ is $\alpha$ upper semi-continuous at $x \in S(X)$, if and only if $\partial\|\cdot\|$ is $\alpha$ upper semi-continuous at $x \in S(X)$. Suppose $\partial\|\cdot\|$ is $\alpha$ upper semi-continuous at $x \in S(X)$, and $\varepsilon>0$ is given. Now since $\partial\|\cdot\|$ is $\alpha$ upper semi-continuous at $x$ there exists a $0<\delta<\varepsilon / 4$ such that $\alpha(\partial\|B(x, \delta)\|)<\varepsilon / 2$. By Proposition 2.1 we have that $S\left(B\left(X^{*}\right), \widehat{x}, \delta^{2}\right) \subseteq \partial\|B(x, \delta)\|+\delta B\left(X^{*}\right)$ and so $\alpha\left(S\left(B\left(X^{*}\right), \widehat{x}, \delta^{2}\right)\right) \leqslant$ $\alpha\left(\partial\|B(x, \delta)\|+\delta B\left(X^{*}\right)\right) \leqslant \alpha(\partial\|B(x, \delta)\|)+\delta \alpha\left(B\left(X^{*}\right)\right)<\varepsilon / 2+\varepsilon / 2=\varepsilon$.

Conversely, if $B\left(X^{*}\right)$ has property $\alpha$ for $\widehat{x} \in S(\widehat{X})$, then given $\varepsilon>0$, there exists a $\delta>0$ such that $\alpha\left(S\left(B\left(X^{*}\right), \widehat{x}, \delta\right)\right)<\varepsilon$. Now, $\partial\|x\| \subseteq S\left(B\left(X^{*}\right), \widehat{x}, \delta\right)$ so by the weak* upper semi-continuity of $\partial\|\cdot\|$ there exists an open neighbourhood $U$ of $x$ such that $\partial\|U\| \subseteq S\left(B\left(X^{*}\right), \widehat{x}, \delta\right)$. So clearly $\alpha(\partial\|U\|)<\varepsilon$.

Corollary 2.3. For a Banach space $(X,\|\cdot\|)$, the dual ball $B\left(X^{*}\right)$ has weak* property $\alpha$ if and only if the duality mapping $x \rightarrow D(x)$ from $S(X)$ into subsets of $S\left(X^{*}\right)$ is a upper semi-continuous on $S(X)$.

For a Banach space $(X,\|\cdot\|)$ we say that the dual norm has the weak $k^{*}$ drop property if for each closed set $C$ in $X^{*}$ for which there exists some $x \in S(X)$ such that $f(x)>1$ for all $f$ in $C$, we have that there exists an element $g \in C$ such that $D\left(g, B\left(X^{*}\right)\right) \cap C=$ $\{g\}$.

We call a sequence $\left\{x_{n}\right\}_{n=1}^{\infty}$ a stream if for each $n \in \mathbb{N}, x_{n+1} \in D\left(x_{n}, B(X)\right) \backslash$ $B(X)$.

THEOREM 2.4. Let $(X,\|\cdot\|)$ be a Banach space. The dual norm $\|\cdot\|$ has the weak $^{*}$ drop property if and only if each stream $\left\{f_{n}\right\}_{n=1}^{\infty}$ in $X^{*}$ where there exists some $x \in S(X)$ such that $f_{n}(x)>1$ for all $n \in \mathbb{N}$, contains a convergent subsequence.

Proof: For the purpose of obtaining a contradiction, assume that the dual norm does not have the weak ${ }^{*}$ drop property. This means that there is a non-empty closed set $C$ in $X^{*}$ and an $x \in S(X)$ with $f(x)>1$ for all $f \in C$, such that for each $g \in C$

$$
\inf \left\{\|f\|: f \in C \cap D\left(g, B\left(X^{*}\right)\right)\right\}=1 .
$$

Indeed, if (1) does not hold, that is, if $r \equiv \inf \left\{\|f\|: f \in C \cap D\left(g, B\left(X^{*}\right)\right)\right\}>1$ then using Danes' classical drop theorem we deduce that there is some $g_{0} \in C \cap D\left(g, B\left(X^{*}\right)\right)$ such that

$$
\left\{g_{0}\right\}=D\left(g_{0}, B\left(X^{*}\right)\right) \cap\left(C \cap D\left(g, B\left(X^{*}\right)\right)\right)=D\left(g_{0}, B\left(X^{*}\right)\right) \cap C .
$$

So by (1) we can choose a stream $\left\{f_{n}\right\}_{n=1}^{\infty}$ in $C$ such that $\lim _{n \rightarrow \infty}\left\|f_{n}\right\|=1$ and $f_{n}(x)>1$ for all $n \in \mathbb{N}$. So by the hypothesis, the stream $\left\{f_{n}\right\}_{n=1}^{\infty}$ contains a convergent 
subsequence $\left\{f_{n_{k}}\right\}_{k=1}^{\infty}$ which converges to $f_{0}$ say, and $\left\|f_{0}\right\|=\left\|\lim _{k \rightarrow \infty} f_{n_{k}}\right\|=\lim _{k \rightarrow \infty}\left\|f_{n_{k}}\right\|=$ 1. Hence $f_{0} \in B\left(X^{*}\right)$. On the other hand, the set $C$ is closed and so is $f_{0} \in C$. This is a contradiction since $B\left(X^{*}\right) \cap C=\emptyset$.

Conversely, suppose that there is a stream $\left\{f_{n}\right\}_{n=1}^{\infty}$ in $X^{*}$ for which there is an $x \in S(X)$ such that $f_{n}(x)>1$ for all $n \in \mathbb{N}$, which contains no convergent subsequences. This means that the set $C \equiv\left\{f_{n}: n \in \mathbb{N}\right\}$ is closed. But clearly for each $n \in \mathbb{N}, f_{n+1} \in D\left(f_{n}, B\left(X^{*}\right)\right) \cap C$, so the dual norm does not have the weak ${ }^{*}$ drop property.

Theorem 2.5. Let $(X,\|\cdot\|)$ be a Banach space. Suppose that there is an $x \in$ $S(X)$ such that the Kuratowski index of non-compactness of the set $G_{e} \equiv\{f: f \in$ $\left.B\left(X^{*}\right), f(x) \geqslant 1-\varepsilon\right\}$ does not tend to zero as $\varepsilon \rightarrow 0$, that is, $\lim _{\varepsilon \rightarrow 0} \alpha\left(G_{\varepsilon}\right)>0$. Then the dual norm does not have the weak* drop property.

Proof: It is easy to observe that for each $\varepsilon>0$ and each finite dimensional subspace $M$ of $X^{*}$

$$
r \equiv \sup _{f \in G_{\varepsilon}}\left(\inf _{g \in M}\|f-g\|\right) \geqslant \frac{1}{2} \alpha\left(G_{\varepsilon}\right) \geqslant \frac{1}{2} \inf _{\varepsilon>0} \alpha\left(G_{\varepsilon}\right)>0
$$

since $G_{e} \subseteq M \cap(1+r) B\left(X^{*}\right)+r B\left(X^{*}\right)$, and so $\alpha\left(G_{e}\right) \leqslant \alpha\left(M \cap(1+r) B\left(X^{*}\right)\right)+$ $r \alpha\left(B\left(X^{*}\right)\right) \leqslant 2 r$. Let $\delta$ be an arbitrary positive number smaller than $1 / 2 \inf _{e} G_{e}$. Now we shall construct by induction a sequence $\left\{f_{0}, f_{1}, \ldots, f_{n}, \ldots\right\}$ such that

$$
f_{n}(x)>1
$$

and

$$
\inf \left\{\left\|f_{n}-g\right\|: g \in \operatorname{sp}\left\{f_{0}, f_{1}, f_{2}, \ldots, f_{n-1}\right\}\right\}>\frac{\delta}{2} .
$$

Suppose that the elements $\left\{f_{0}, f_{1}, \ldots, f_{n}\right\}$ satisfying (2i) and (3i) for $i \in\{1,2, \ldots, n\}$ have been constructed. Take $0<\varepsilon<f_{n}(x)-1$, and let $\bar{f}_{n+1}$ be an arbitrary element of $G_{e}$ such that $\inf \left\{\left\|\bar{f}_{n+1}-g\right\|: g \in \operatorname{sp}\left\{f_{0}, f_{1}, \ldots, f_{n}\right\}\right\}>\delta$. Such an element exists by (1), and by the definition of $\delta$. Let $f_{n+1} \equiv(1 / 2) f_{n}+(1 / 2) \bar{f}_{n+1}$. Then by the choice of $\varepsilon$

$$
f_{n+1}(x)=\frac{1}{2} f_{n}(x)+\frac{1}{2} \bar{f}_{n+1}(x) \geqslant \frac{1}{2} f_{n}(x)+\frac{1}{2}(1-\varepsilon)>1 .
$$

Moreover,

$$
\begin{aligned}
& \inf \left\{\left\|f_{n+1}-g\right\|: g \in \operatorname{sp}\left\{f_{0}, f_{1}, \ldots, f_{n}\right\}\right\} \\
& \quad=\frac{1}{2} \inf \left\{\left\|\bar{f}_{n+1}-g\right\|: g \in \operatorname{sp}\left\{f_{0}, f_{1}, \ldots, f_{n}\right\}\right\}>\frac{\delta}{2}
\end{aligned}
$$


by the choice of $\delta$. Let $C \equiv\left\{f_{0}, f_{1}, \ldots, f_{n}, \ldots\right\}$, then $C$ is closed and $f_{n}(x)>1$ for each $n \in \mathbb{N}$. Further, by the construction $f_{n+1} \in D\left(f_{n}, B\left(X^{*}\right)\right) \cap C$, and hence the dual norm does not have the weak* drop property. So, we conclude that if the dual norm has the weak ${ }^{*}$ drop property then $B\left(X^{*}\right)$ has the weak ${ }^{*}$ property $\alpha$.

In the following theorem we prove the converse of this.

Theorem 2.6. Let $(X,\|\cdot\|)$ be a Banach space. If the dual ball $B\left(X^{*}\right)$ has the weak $^{*}$ property $\alpha$ then the dual norm has the weak ${ }^{*}$ drop property.

ProOF: Let $\left\{f_{n}\right\}_{n=1}^{\infty}$ be an eventually non-constant stream in $X^{*}$ with $f_{n}(x)>1$ for all $n \in \mathbb{N}$, for some fixed $x \in S(X)$. Suppose first that $r \equiv \inf \left\{\left\|f_{n}\right\|: n \in \mathbb{N}\right\}>1$. If $\left\{f_{n}\right\}_{n=1}^{\infty}$ does not have a convergent subsequence then $\left\{f_{n}: n \in \mathbb{N}\right\}$ is a closed set at a positive distance from $B\left(X^{*}\right)$. By Danes' classical Drop Theorem, there exists a point $f \in\left\{f_{n}: n \in \mathbb{N}\right\}$ such that $D\left(f, B\left(X^{*}\right)\right) \cap\left\{f_{n}: n \in \mathbb{N}\right\}=\{f\}$, which is impossible for such a stream. Thus, $\left\{f_{n}\right\}_{n=1}^{\infty}$ has a convergent subsequence. On the other hand, if $\inf \left\{\left\|f_{n}\right\|: n \in \mathbb{N}\right\}=1$ then the sequence $\left\{\left\|f_{n}\right\|\right\}_{n=1}^{\infty}$ converges to 1 since $\left\|f_{n+1}\right\| \leqslant\left\|f_{n}\right\|$ for all $n \in \mathbb{N}$. Let $f_{0}$ be a weak ${ }^{*}$ cluster point of $\left\{f_{n}\right\}_{n=1}^{\infty}$. Now, since the dual norm is weak ${ }^{*}$ lower semi-continuous $\left\|f_{0}\right\| \leqslant 1$, but $f_{0} \in\{f \in$ $\left.X^{*}: f(x) \geqslant 1\right\}$, so $f_{0}(x)=\left\|f_{0}\right\|=1$. We note that $f_{0}$ is also a weak cluster point of the sequence $\left\{f_{n} /\left\|f_{n}\right\|\right\}_{n=1}^{\infty}$. Now, for each $k \in \mathbb{N}$ choose $g_{n_{k}} \in\left\{f_{n} /\left\|f_{n}\right\|: n \in \mathbb{N}\right\}$ such that $g_{n_{k}} \in S\left(B\left(X^{*}\right), \widehat{x}, 1 / k\right)$ and $g_{n_{k}} \notin\left\{g_{n_{1}}, g_{n_{2}}, \ldots, g_{n_{k-1}}\right\}$. We see then that $\alpha\left(\left\{g_{n_{k}}: k \in \mathbb{N}\right\}\right)=0$, and therefore the set $\overline{\left\{g_{n_{k}}: n \in \mathbb{N}\right\}}$ is compact. Thus $\left\{g_{n_{k}}\right\}_{k=1}^{\infty}$ contains a convergent subsequence $\left\{g_{n_{k_{\ell}}}\right\}_{\ell=1}^{\infty}$ which converges to $h_{0}$ say, and $\left\|h_{0}\right\|=$ $h(x)=1$. Hence,

$$
\lim _{\ell \rightarrow \infty} f_{n_{k_{\ell}}}=\lim _{\ell \rightarrow \infty}\left\|f_{n_{k_{\ell}}}\right\| \cdot g_{n_{k_{\ell}}}=\lim _{\ell \rightarrow \infty}\left\|f_{n_{k_{\ell}}}\right\| \cdot \lim _{\ell \rightarrow \infty} g_{n_{k_{\ell}}}=h_{0} .
$$

So the dual norm has the weak * drop property.

ThEOREM 2.7. For a Banach space $(X,\|\cdot\|)$, the following are equivalent:

1. The duality mapping $x \rightarrow D(x)$ is a norm cusco on $S(X)$.

2. The duality mapping $x \rightarrow D(x)$ is a upper semi-continuous on $S(X)$.

3. The dual ball $B\left(X^{*}\right)$ has weak property $\alpha$.

4. Every stream $\left\{f_{n}\right\}_{n=1}^{\infty}$ in $X^{*}$ such that $f_{n}(x)>1$ for all $n \in \mathbb{N}$, for some fixed $x \in S(X)$, contains a convergent subsequence.

5. The dual norm has the weak drop property.

We see then that every Banach space $X$ whose dual norm has the drop property has the weak * drop property. However it is known, see [4], that the duality mapping $x \rightarrow D(x)$ on $S\left(c_{0}(\Gamma)\right)$ is a upper semi-continuous on $S\left(c_{0}(\Gamma)\right)$. Hence $\ell_{1}(\Gamma)$ with its usual norm has the weak * drop property, but not the drop property, when $\Gamma$ is an infinite set, as $\ell_{1}(\Gamma)$ is not reflexive in this case. 
Now in [5, Theorem 3] Montesinos showed that if the closed unit ball $B(X)$ has property $\alpha$ for each $f \in S\left(X^{*}\right)$ then the norm on $X$ has the drop property. So it follows from Theorem 2.7 Part 3 that a reflexive space whose dual norm has the weak* drop property has the drop property.

We shall now show that the norm $\|\cdot\|$ on a reflexive Banach space $X$ has the drop property if and only if every separable quotient norm has the drop property. We shall proceed by showing that the duality mapping $f \rightarrow D(f)$ from $S\left(X^{*}\right)$ into subsets of $S\left(X^{* *}\right)$ is $\alpha$ upper semi-continuous on $S\left(X^{*}\right)$ if and only if each separable quotient norm has the drop property.

THEOREM 2.8. Let $E$ be a non-empty subset of an open convex subset $A$ of a Banach space $X$. Consider $\phi$ a continuous convex function on $A$. Then $\partial \phi$ is $\alpha$ upper semi-continuous on $E$ if and only if $\partial\left(\left.\phi\right|_{Y}\right)$ is a upper semi-continuous on $E \cap Y$ for every closed separable subspace $Y$ of $X$ such that $E \cap Y \neq \emptyset$.

Proof: Clearly if $\partial f$ is $\alpha$ upper semi-continuous on $E$ then $\partial\left(\left.\phi\right|_{Y}\right)$ is $\alpha$ upper semi-continuous on $E \cap Y$ for every separable subspace $Y$ of $X$. Since $\partial\left(\left.\phi\right|_{Y}\right)=z^{*} \partial \phi$, where $\imath$ is the inclusion map from $Y$ into $X$ and $\imath^{*}$ is its conjugate map from $X^{*}$ into $Y^{*}$.

Conversely suppose that $\partial f$ is not $\alpha$ upper semi-continuous on $E$. Then there is some $x \in E$ and $r>0$ such that $\alpha(\partial \phi(B(x, 1 / n) \cap E))>2 r$ for every $n \in \mathbb{N}$. For a fixed $n \in \mathbb{N}$, we can choose two sequences $\left\{f_{k}^{n}\right\}_{k=1}^{\infty} \subseteq X^{*}$ and $\left\{x_{k}^{n}\right\}_{k=1}^{\infty} \subseteq B(x, 1 / n) \cap E$ such that $\bigcup_{k=1}^{\infty}\left\{f_{k}^{n}\right\} \subseteq \partial \phi(B(x, 1 / n) \cap E)$ and $\left\|f_{r}^{n}-f_{e}^{n}\right\|>r$ if $r \neq s$ with $f_{k}^{n} \in \partial \phi\left(x_{k}^{n}\right)$ for all $k \in \mathbb{N}$, (see Proposition 1.3 Part 2). Now, for a fixed $k \in \mathbb{N}$ we can choose another sequence $\left\{y_{j}^{n k}\right\}_{j=1}^{\infty}$ contained in $S(X)$ such that $\left(f_{k}^{n}-f_{j}^{n}\right)\left(y_{j}^{n k}\right)>r$, if $j \neq k$. Let $Y \equiv \bar{s} \bar{p}\left\{y_{j}^{n k}, x_{k}^{n}, x: n, k, j \in \mathbb{N}\right\}$. Clearly $Y$ is separable and $E \cap Y \neq \emptyset$. We shall now show that $\partial\left(\left.\phi\right|_{Y}\right)$ is not $\alpha$ upper semi-continuous at $x \in E \cap Y$. For $n \in \mathbb{N}$,

$$
\begin{aligned}
\imath^{*}\left(\partial \phi\left(\imath\left(B\left(x, \frac{1}{n}\right) \cap E \cap Y\right)\right)\right) & \supseteq \imath^{*}\left(\partial \phi\left(\bigcup_{k=1}^{\infty}\left\{x_{k}^{n}\right\}\right)\right)=\imath^{*}\left(\bigcup_{k=1}^{\infty} \partial \phi\left(x_{k}^{n}\right)\right) \\
& \supseteq \imath^{*}\left(\bigcup_{k=1}^{\infty}\left\{f_{k}^{n}\right\}\right)=\bigcup_{k=1}^{\infty}\left\{\imath^{*}\left(f_{k}^{n}\right)\right\}
\end{aligned}
$$

but $\left\|\imath^{*}\left(f_{r}^{n}\right)-\imath^{*}\left(f_{a}^{n}\right)\right\| \geqslant\left(f_{r}^{n}-f_{?}^{n}\right)\left(y_{s}^{n r}\right)>r$ if $r \neq s$. So from Proposition 1.3 Part 1, $\alpha(\partial(\phi \mid Y)(B(x, 1 / n) \cap E) \cap Y) \geqslant r$. Hence $\partial(\phi \mid Y)$ is not $\alpha$ upper semi-continuous on $E$.

Corollary 2.9. Let $(X,\|\cdot\|)$ be a reflexive Banach space. Then the norm on $X$ has the drop property if and only if the norm on every separable quotient of $X$ has the drop property. 
Proof: Suppose that every separable quotient norm has the drop property. We shall now proceed by showing that the duality mapping $f \rightarrow D(f)$ on $S\left(X^{*}\right)$ is $\alpha$ upper semi-continuous on $S\left(X^{*}\right)$. Let $F$ be a closed separable subspace of $X^{*}$, then $F$ is reflexive. Furthermore, $F^{*}$ is isometrically isomorphic to $\left(X / F^{\perp}\right)$ where $F^{\perp} \equiv$ $\{x \in X: f(x)=0$ for all $f$ in $F\}$. Therefore, the duality mapping $f \rightarrow D(f)$ from $S(F)$ into subsets of $S\left(F^{*}\right)$ is $\alpha$ upper semi-continuous on $S(F)$. Hence by Theorem 2.8 , the duality mapping is $\alpha$ upper semi-continuous on $S\left(X^{*}\right)$, and so the norm $X$ has the drop property.

The converse follows from the facts that the dual of $(X / F)$ is isometrically isomorphic to $F^{\perp}$, where $F^{\perp} \equiv\left\{f \in X^{*}: f(x)=0\right.$ for all $x$ in $\left.F\right\}$, and that the duality mapping $f \rightarrow D(f)$ from $S\left(F^{\perp}\right)$ into subsets of $S\left(\left(F^{\perp}\right)^{*}\right)$ is $\alpha$ upper semi-continuous on $S\left(F^{\perp}\right)$, (see Theorem 2.8).

\section{References}

[1] J. Banas and K. Goebel, Measures of non-compactness in Banach spaces (Marcel Dekker, New York and Basel, 1980).

[2] J. Danes̃, 'A geometric theorem useful in non-linear functional analysis', Boll. Un. Math. Ital. 6 (1972), 369-372.

[3] J.R. Giles, B. Sims and A.C. Yorke, 'On the drop and weak drop properties for a Banach space', Bull. Austral. Math. Soc. 41 (1990), 503-507.

[4] J.R. Giles and W.B. Moors, 'A continuity property related to Kuratowski's index of non-compactness, its relevance to the drop property and its implications for differentiability theory', J. Math. Anal. Appl. (to appear).

[5] V. Montesinos, 'Drop property equals reflexivity', Studia Math. 87 (1987), 93-100.

[6] W.B. Moors, 'A continuity property related to an index on non-separability and its applications', Bull. Austral. Math. Soc. 46 (1992) (to appear).

[7] S. Rolewicz, 'On drop property', Studia Math. 85 (1987), 27-35.

Department of Mathematics

Auckland University

Private Bag, Auckland

New Zealand 